\title{
Research on a hybrid map matching algorithm for Global Navigation Satellite System based train positioning
}

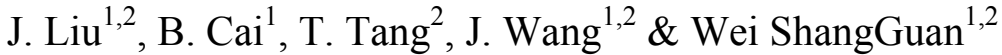 \\ ${ }^{I}$ School of Electronics and Information Engineering, \\ Beijing Jiaotong University, China \\ ${ }^{2}$ State Key Laboratory of Rail Traffic Control and Safety, \\ Beijing Jiaotong University, China
}

\begin{abstract}
GNSS has been proved to have great potential for Safety-of-Life critical rail applications, particularly the train control technique and railway signalling. In the GNSS based train positioning scheme, although with the aid of inertial sensors (e.g. the odometer, gyro, accelerator and Doppler radar) some systematic and random errors could be reduced or limited by an appropriate measuring method and data fusion filtering, it is significant to improve and guarantee the positioning precision and integrity performance by using the map matching (MM) technique in a cost effective way. In this paper, the structure of an electrical track map database is designed according to the requirements of precision and efficiency, the architecture of a GNSS based train positioning system integrating INS sensors is introduced, and a novel hybrid map matching algorithm is proposed, in which the determined train position is the integration of the position solution from multi-sensor fusion, the identification of the similarity or matching probability, and heading validation, with different track map levels. As the "point-to-curve" and "point-to-point" matching strategy are adopted with the provided feature of track map data, the adaptive performance and completeness of the map matching algorithm is guaranteed and improved. A field test in the Qinghai-Tibet line demonstrates that the proposed algorithm earns high position decision accuracy and integrity with simple implementation, which is of great practical value to precise train control and railway signalling.
\end{abstract}

Keywords: map-matching, train positioning, integrated positioning, GNSS, INS, track map database, similarity, train control. 


\section{Introduction}

The fast developing GNSS (Global Navigation Satellite System), including U.S. GPS, European Galileo, Russian GLONASS and Compass in China, will play a more important role in railway transport, especially the signalling and traffic control. The integration of satellite navigation systems and the ERTMS/ETCS will bring great benefits to both corridor and regional low density lines. Within recent years, a number of R\&D determination projects based on GNSS have been carried out world-wide, such as ATCS, ARES, PTS and NAJPTC in North America and APOLO, ECORAIL, LOCOPROL/LOCOLOC, RUNE and GADEROS in Europe [1, 2].

China has been developing the modern train control system, named CTCS (China Train Control System), and has reached the CTCS level 3 [3]. With the implementation of the next generation satellite system Compass, there will be a high demand for the GNSS technique for safety related railway applications in China.

The position of the train is the core function of all the railway operations. Quite different demands on an on-board GNSS based train positioning system are required by safety related applications, mainly those concerning signalling and train control, and one important aspect of them is to develop the positioning system as precisely and cost-effectively as possible [4-6].

Due to the disadvantages of single sensor configuration for train positioning systems, a multi-sensor based structure has been an inevitable trend to improve the performance of accuracy, reliability and integrity. In the position sensing and measuring process, there must be some systematic and random noise to increase the deviation between the real train position in the Map Set Space and the practical measurements in Measuring Space, in which the final measuring error is the combination of sensor behaving error and the stochastic interference (Fig. 1).

With the analysis of train position sensing, then the train positioning process, which is aiming at the integrity, accuracy and reliability, could be divided into

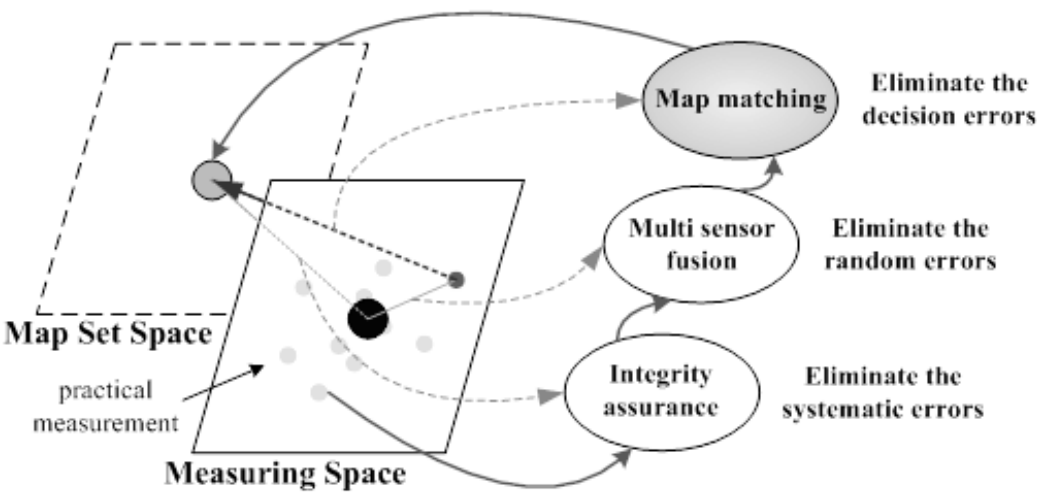

Figure 1: Architecture of the train positioning process. 
three main steps to return to the original position state constrained by the rail track map. The three steps, integrity assurance, multi-sensor fusion and map matching, are designed to solve the systematic, random and errors in position decisions.

Among the three steps, the map matching calculation, which provides a link by integrating positioning data with spatial track map data to identify the correct geographical position and the track that the train is moving on, is the key component to improve and realize the required performance index. In recent years, there have been a lot of map matching algorithms developed for GNSS based transport applications, and those approaches can be categorised into four groups: geometric, topological, probabilistic and other advanced techniques, which have been introduced and detailed in [7]. For the one-dimensional character of rail trains and the switch based topological structure of rail tracks, the geometric way is most direct approach to realize the matching process, such as the vertical projection from the positioning fix to the connection between candidate track points [8], correlating the angular rate extracted from the map database to the corresponding measurements [9]. In order to be capable of supporting the requirements of various operation conditions, the integrity, adaptive ability and computational efficiency should be concerned in the design of the map matching algorithm. In this paper, based on the analysis of rail track map structure and multi sensor integration, a hybrid map matching algorithm is proposed with similarity extraction, point matching and the heading validation in different map levels, and the algorithm can be implemented into various train positioning solutions.

\section{Track map database for train positioning}

Map-matching not only enables the physical location of the train to be identified, but also improves the positioning accuracy, if precise track map data is available. There must be some kind of error on the track map for the inadequate measuring means and uncertainties derived from the generation process. So the precision of track map is a crucial factor to the map matching approaches.

The railway track map is composed of rail track lines and the rail equipments along the lines. Measurements of the track map are always obtained by static measurement in a long period at the key points (such as the switch and signal controller) and dynamic operation along the centre of track lines. With post processing of track position measuring data and corresponding completeness, the map could be expressed or described by the track map database, combining discrete track line points and the attribute data.

As the cost of the track map database depends on the accuracy it holds (i.e. higher track database accuracy leads to high complexity and expense), according to different operation conditions, the map is generated at three levels separately, which is as shown in Fig. 2.

For level 1, there are only position and attribute data of points of interesting collected, which describe the most significant information of the track lines, with high accuracy measuring, such as switch, signal controller and insulation section. 
However, the description of the rail track in this level is a low resolution strategy, and the precision performance could be compensated by the cost effectiveness.

For level 2, except the key track points in level 1, some characteristic points of track curve are extracted from raw GNSS track measurements by certain curve feature extraction and data reducing algorithm (i.e. Douglas-Peucker algorithm), then the precision of the track description will be improved to fulfil the matching requirements.

For level 3, regardless of the cost of track map building, the detailed curve feature information of rail tracks are introduced by interpolating the key points and characteristic points in level 1 and 2 as the cubic B-spline principle. A factor of precision is employed to constrain the uniform interpolation and evaluate the point matching performance which will be presented in following chapters.

\section{GNSS based integrated train positioning}

Train positioning system could get lots of benefit (i.e. lower cost, better precision and time-space coverage) from the application of GNSS, hence GNSS technique has been integrated into some current train control and positioning systems in the form of core position sensor or the virtual balise. But there are still some safety risks for the satellite based system, such as the limited SIS availability, multipath effect, Signal-In-Space verification and the electromagnetic interference, so the GNSS-only strategy for train positioning cannot cover the performance indices completely, then the multi-sensor integration is found an effective approach.

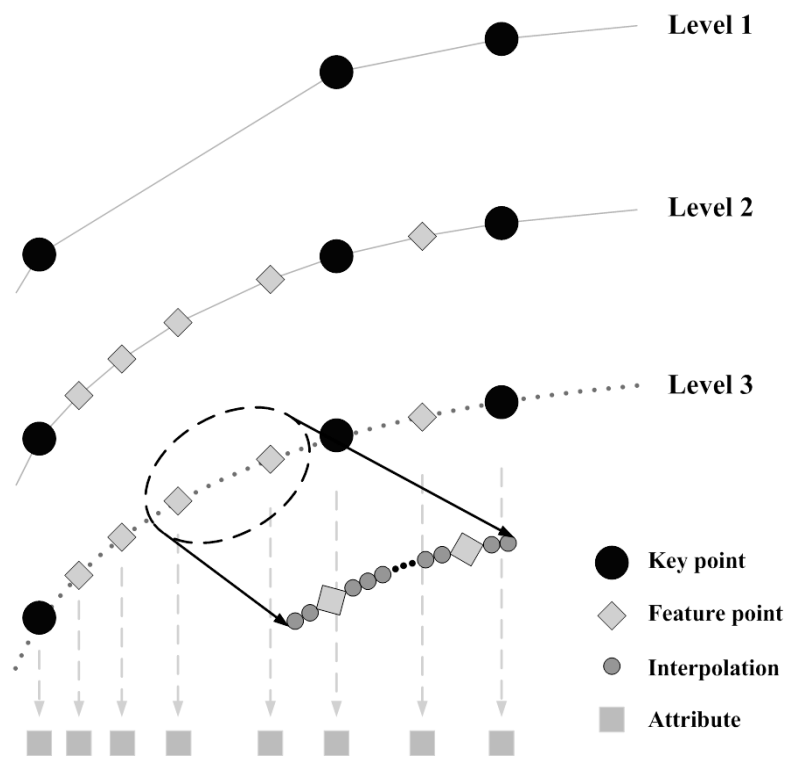

Figure 2: $\quad$ Structure of the track map for train positioning. 
It is evident that railway GNSS / INS (Inertial Navigation System) based safety of life applications acquire highly reliable and accurate data provided by onboard sensors. The core function of position (include the geographic location, velocity and headings of a train, in a broader sense) could be realized by fusion of data from GNSS receiver and INS sensors, which can be simply described as follows: odometer, accelerometer and Doppler radar for distance calculation and validation, and gyroscope for gyro-odometry for routing detection on switch [11].

As an important component of train control system, multi-sensor integrated positioning system could be introduced to current train control systems in many ways, depending on the application level, interoperation capability and operation context (high speed lines, low density lines, etc). With current odometer based scheme in train control, two main integration approaches are as follow:

I. GNSS/INS enhances the odometer, in which the GNSS/INS based system is taken as a complement to enhance the position determination function of the current existing position sensors (the odometer with calibration from balise), thus the integration could be realized without breaking the current configuration and interoperation of the train control system.

II. GNSS/INS substitutes the odometer, in which GNSS/INS based system is employed to realize the whole function of position determination, replacing the current odometer based positioning solution, then that will be more independent and flexible for the integration to innovate the train control architecture.

In practical implementation, approach I is more feasible for configuration compatibility. The architecture of GNSS based integrated positioning system in approach I is as Fig. 3.

On-board unit calculate the train position with position sensor data and the cubature Kalman filter, which has been proved an efficient nonlinear data fusion algorithm. The final position is determined and calibrated to the track map which is taken as the absolute reference. In this map matching process, track map is used as another "virtual sensor" in the form of a database, with the architecture as described in former chapter.

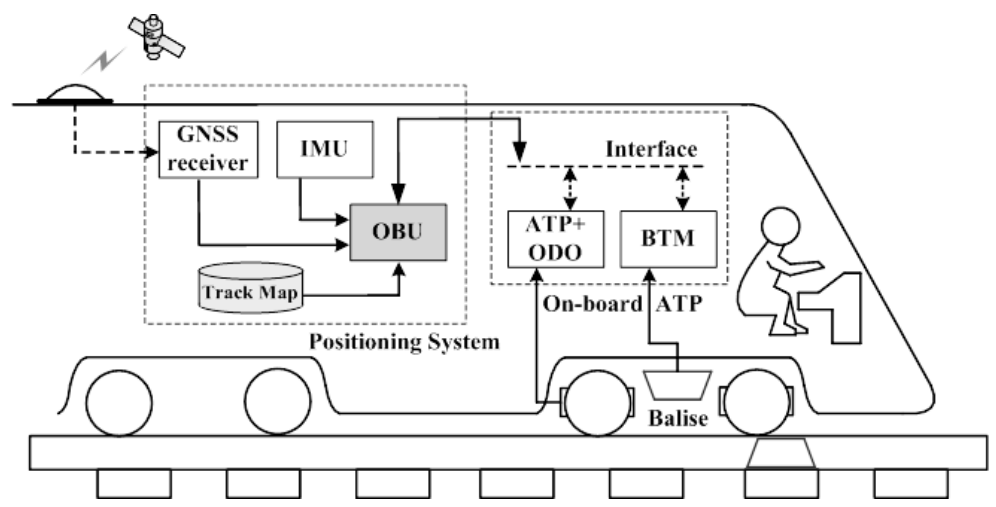

Figure 3: Architecture of GNSS based integrated train positioning. 


\section{Hybrid map matching for position determination}

Map matching is a software algorithm that is used to integrate various position sensors data with map data to give a better position estimate of trains. It plays an important role in the positioning system as it employs digital track map database to improve the accuracy and reliable of positioning system, with the principle that trains can only move along the fixed track lines.

In practical application, based on the discussion of the structure of track map database, map in level 2 and level 3 are feasible in most conditions. As for the different feature of the map levels, the map matching algorithm is designed and tested separately.

To map matching in level 2, as the medium precision map data are provided, the "point-to-curve" strategy is adopted for matching algorithm; the similarity maximization principle is used to obtain the optimal matched position. Assume $\boldsymbol{p}_{f}(k)=\left[\begin{array}{lll}x_{f}(k) & y_{f}(k) & l_{f}(k)\end{array}\right]^{\mathrm{T}}$ is the output of multi-sensor integration at time $k$, where $x_{f}(k)$ and $y_{f}(k)$ are train position in east and north direction, and $l_{f}(k)$ is the travelling distance. $\left\{\boldsymbol{V}_{j}\right\}$ are track line data in level 2, including the key track points and curve characteristic points, and $\boldsymbol{V}_{j}=\left[\begin{array}{lll}x_{j} & y_{j} & l_{j}\end{array}\right]^{\mathrm{T}}$.

The aim of map matching is to determine the matched position $\boldsymbol{p}_{m}(k)$ for the calculated $\boldsymbol{p}_{f}(k)$ with maximal probability and similarity. The hybrid matching algorithm combines the CKF based data fusion with the similarity identification and heading validation. The algorithm could be divided into three key operations:

(1) Obtain the data fusion position.

The cubature Kalman filter (CKF) is a Gaussian approximation to Bayesian filter, with more accurate filtering performance than traditional method and less computational cost [13]. As some inertial sensors' measurements have nonlinear relation with filtering state, the CKF approach is adopted to estimate the position error and compensate the inertial calculation, which is the foundation to match the map database.

(2) Acquire the candidate map segment.

Use the distance between fusion output $\boldsymbol{p}_{f}(k)$ and map position $\left.\left\{\boldsymbol{V}_{j}\right\}\right|_{j=i} ^{i+N}$ to compute the probability of the candidate segment, with a fixed length "window" of $N$ points. The Gaussian function based probability is

$$
p_{c a}(k, j)=\frac{1}{\sqrt{2 \pi} \sigma_{c}} \cdot \exp \left(-\left\|\frac{\boldsymbol{p}_{f}(k)-\boldsymbol{V}_{j}}{h}\right\|^{2}\right)
$$

The most probable extreme point can be determined by

$$
\boldsymbol{M}_{f}(k)=\arg \max _{j} p_{c a}(k, j)
$$


Then the other endpoint of the candidate segment $\boldsymbol{M}_{e}(k)$ could be selected by the nearest strategy with train travelling distance. The curve segment $\overline{\boldsymbol{M}_{f} \boldsymbol{M}_{e}}$ as candidate will be the map data set for matching.

(3) Similarity calculation and identification.

In order to find the matching position $\boldsymbol{P}_{m}(k)$ from segment $\overline{\boldsymbol{M}_{f} \boldsymbol{M}_{e}}$, the similarity is employed to be the decisive factor for the "point-to-curve" solution.

Firstly, initialize the target model with the fusion position and candidate map segment from step (1) and (2). The distance between $\boldsymbol{p}_{f}(k), \boldsymbol{M}_{f}(k)$ and $\boldsymbol{M}_{e}(k)$ in east and north are taken as the feature factors, and the initial target model can be given as

$$
\begin{cases}\hat{q}_{1}=C \cdot \exp \left(\left\|\frac{x_{f}(k)-x_{m f}}{h_{0}}\right\|^{2}\right), & \hat{q}_{2}=C \cdot \exp \left(\left\|\frac{x_{f}(k)-x_{m e}}{h_{0}}\right\|^{2}\right) \\ \hat{q}_{3}=C \cdot \exp \left(\left\|\frac{y_{f}(k)-y_{m f}}{h_{0}}\right\|^{2}\right), & \hat{q}_{4}=C \cdot \exp \left(\left\|\frac{y_{f}(k)-y_{m e}}{h_{0}}\right\|^{2}\right)\end{cases}
$$

where $C$ is the normalization factor, $h_{0}$ is the bandwidth.

Then the candidate target model could be described with the same parameters. For a candidate element $\boldsymbol{P}(n) \in \overline{\boldsymbol{M}_{f} \boldsymbol{M}_{e}}$, the model could be

$$
\begin{cases}\tilde{p}_{1}=C_{p} \cdot \exp \left(\left\|\frac{x_{p}(n)-x_{m f}}{h_{0}}\right\|^{2}\right), & \tilde{p}_{2}=C_{p} \cdot \exp \left(\left\|\frac{x_{p}(n)-x_{m e}}{h_{0}}\right\|^{2}\right) \\ \tilde{p}_{3}=C_{p} \cdot \exp \left(\left\|\frac{y_{p}(n)-y_{m f}}{h_{0}}\right\|^{2}\right), & \tilde{p}_{4}=C_{p} \cdot \exp \left(\left\|\frac{y_{p}(n)-y_{m e}}{h_{0}}\right\|^{2}\right)\end{cases}
$$

Finally, combine the target model and the candidate, the similarity function is proposed for evaluation, which is defined as

$$
\hat{\rho}(n)=\rho[\tilde{p}(n), \hat{q}]=\sum_{i=1}^{4} \sqrt{\tilde{p}_{i}(n) \bullet \hat{q}_{i}}
$$

where the similarity $\hat{\rho} \in[0,1]$, and the larger $\hat{\rho}$ is, the more similar features are identified between fusion position and the candidate model $\boldsymbol{P}(n)$. The matched position can be

$$
\boldsymbol{P}_{m}(k)=\arg \max _{n} \hat{\rho}(n)
$$

The similarity based map matching process in level 2 could be described as the upper graph of Fig 4. 


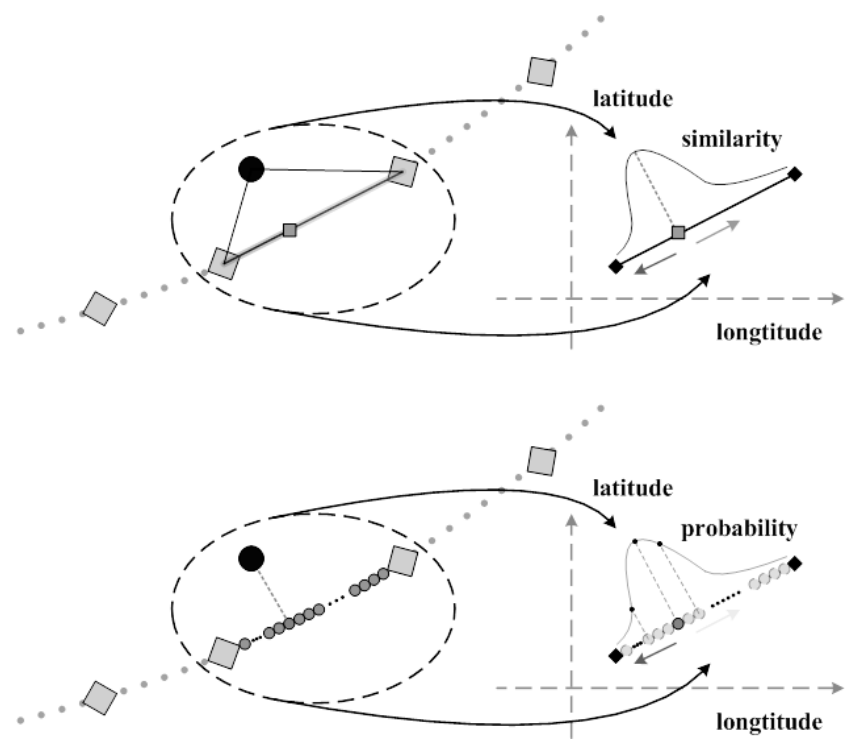

Figure 4: $\quad$ Principle of the map matching algorithm.

(4) Heading validation.

For the validation of $\boldsymbol{P}_{m}(k)$ to be the final matched output, an independent testing method is adopted with the heading of the train. Under the ideal condition, the heading variation of the train trajectory should be the same as that with map matching. In practical train operation environment, interferences and errors lead to some differences to a certain extent. Given a reasonable error threshold, then the map matched position could be validated and the positioning precision and integrity monitoring will be realized simultaneously.

Assume the error threshold is $\xi$, and then the validation could be defined as

$$
\delta h(k)=\left|h_{f}(k)-h_{m}(k)\right| \leq \xi
$$

where the $h_{f}(k)$ and $h_{m}(k)$ are heading at fusion position and the map matched respectively.

To map matching in level 3, where the interpolation map data are available, as the high precision map data are provided with predefined precision factor $\delta d$, which is usually at decimetre level, in order to keep a balance between efficiency of map storage and matching computation, the "point-to-point" strategy is used to realize the map matching.

Map matching process in level 3 has the same step (1), (2) and (4) as that in level 2. Here in the step (3), candidate segment $\overline{\boldsymbol{M}_{f} \boldsymbol{M}_{e}}$ provide corresponding interpolation data set $\left\{\boldsymbol{C}_{j} \mid \overline{\boldsymbol{M}_{f} \boldsymbol{M}_{e}}\right\}$ for the determination "which" point would be chosen as the matching result. 
Similar to the acquisition of the most probable extreme point of candidate segment, the matching operation is based on the maximum probability principle, which is as shown in lower graph of Fig 4, where $\boldsymbol{P}_{m}(k)$ satisfies

$$
\boldsymbol{P}_{m}(k)=\arg \max _{j}\left(\omega \cdot \exp \left(-\left\|\frac{\boldsymbol{P}_{f}(k)-\boldsymbol{C}_{j}}{h}\right\|^{2}\right)\right)
$$

From the detailed analysis of the map matching in different map levels, the whole hybrid map matching process could be unified into one flow diagram, which is as shown in Fig 5.

In the unified process, the judgement of "Interpolation data available" is the key step to vary the different map level based matching strategies. Only when the heading validation is successful, the calculated matching position would be used for output, and evaluation of the positioning precision and integrity.

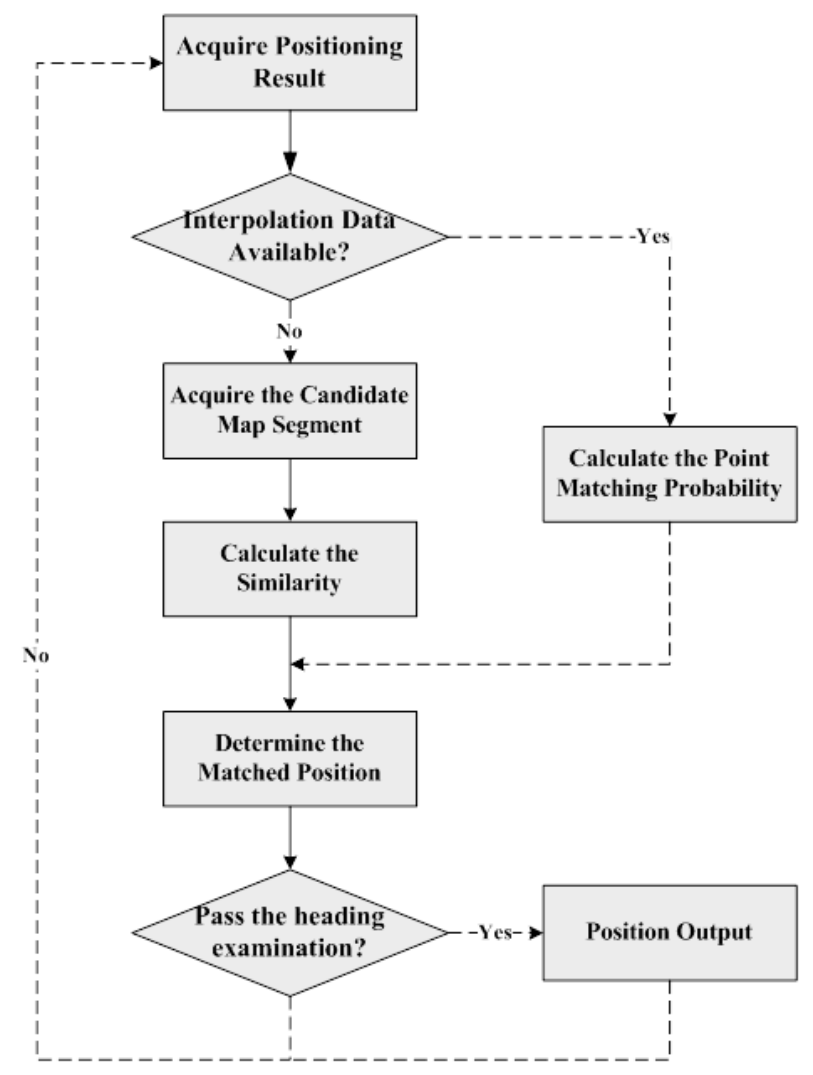

Figure 5: $\quad$ Flow of the hybrid map matching process. 


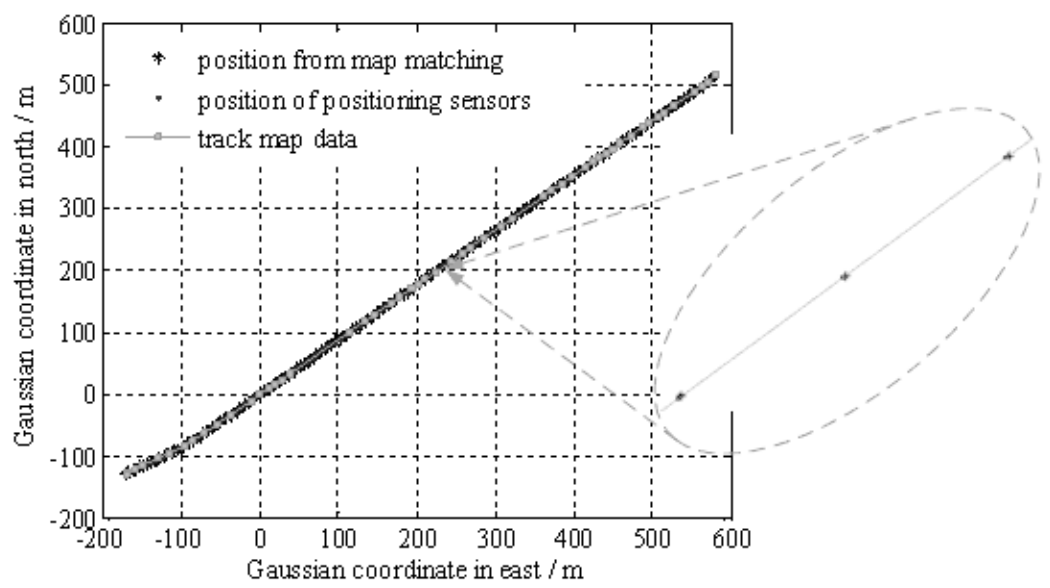

Figure 6: $\quad$ Map matching compared with sensors and track map.

\section{Field test and validation}

In order to validate the performance of proposed hybrid map matching algorithm in this paper, field tests have been conducted with electrical track map generated from Qinghai-Tibet rail line in June 2009.

A high precision GNSS receiver is employed to collect position measurements along practical tracks or at the points of interest, and then the track map levels 1-3 are generated with strict criteria. Extensive map validation collections are also taken to test and validate the proposed map matching algorithm.

Fig 6 shows the map matching results compared with the positioning sensor fusion and track map data from a station, where all the position coordinates have been shifting transformed. As is shown in the elliptical area, actually the position from sensors are even close to the track lines, however, the map matching isolate the error and further improves the positioning accuracy.

Take travelling distance as the one-dimensional map description, Fig 7 shows how the similarity distributes and varies in the map matching process with 193 frame sensor measurements.

The maximum similarity of every epoch indicates the matched position from the hybrid map matching algorithm, and from the similarity based determination process. It can be concluded that the hybrid algorithm preserves the advantages of both the geometric and probabilistic characters. With the "point-to-curve" or "point-to-point" strategy, the "longitude-latitude-altitude-similarity /probability" character space architecture is constructed and applied to every received sensor data frame, and then the position matching is the dynamic form of the space. As the results of the field test and validation shown, the proposed hybrid algorithm earns a high adaptive ability for the track map level to achieve high precision, reliability and completeness in GNSS based train positioning. 


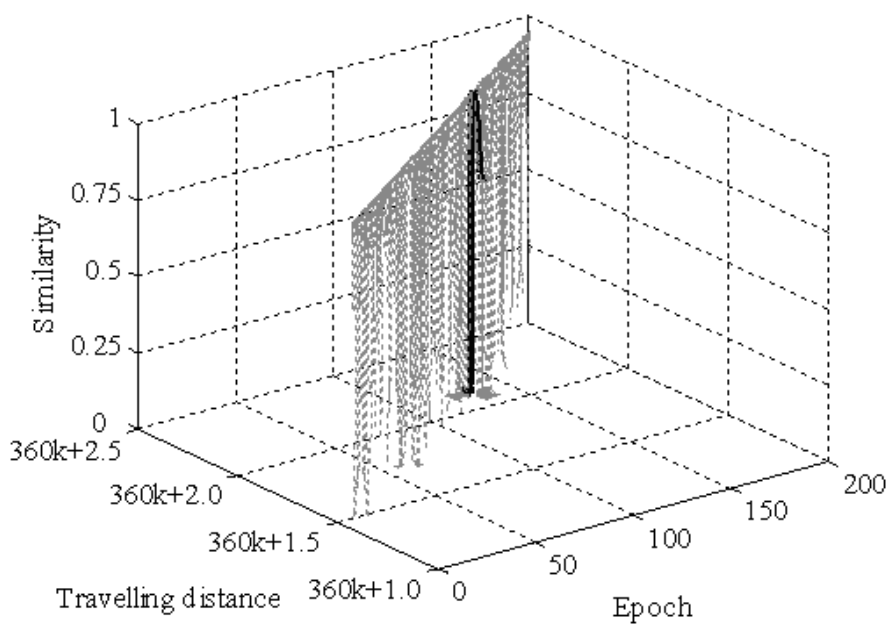

Figure 7: $\quad$ Similarity variety in the map matching process.

\section{Conclusion}

In this paper, an approach for train position determination with an electronic rail track map is demonstrated, with a novel map matching algorithm proposed for GNSS based train positioning. Based on the architecture analysis of track map database and the GNSS based train positioning system, a hybrid map matching algorithm is proposed with four key steps, where the judgement for map interpolation data is used to distinguish matching strategies in different map level, and the heading validation for correction assurance. The proposed approach holds high precision and computational efficiency, and field tests validated the conclusions, including that the accurate sensor integration and precise track map data are also crucial for realization of GNSS based train positioning and train control.

\section{Acknowledgements}

This work was supported by National Natural Science Foundation of China (No.60736047, 60634010, 60870016), and the Fundamental Research Funds for the Central Universities (No.2009YJS020).

\section{References}

[1] Filip A., Bazant L., Taufer J., Maixner V., Mocek H., Train-borne position integrity monitoring for GNSS/INS based signalling, International Symposium on Speed-up and Service Technology for Railway and Maglev Systems '2003, Tokyo, Japan, 2003, pp. 88-93. 
[2] Polivka A., Filip A., Satellite-Based Positioning for CBTC, the 2nd international conference "Reliability, safety and diagnostics of transport structures and means 2005", Pardubice, Czech Republic, 2005.

[3] Cai B., Shangguan W., Li X., Wang J., Research on supporting technology for simulation CTCS-3 based on multi-resolution modelling, Journal of Beijing Jiaotong University, vol. 34, no. 2, 2010, pp. 5-10.

[4] Simsky A., Wilms F., Franckart J-P., GNSS-based failsafe train positioning system for low-density traffic lines based on one-dimensional positioning algorithm, 2nd ESA Workshop on Satellite Navigation User Equipment Technologies, Noordwijk, Netherlands, 2004, pp. 1-8.

[5] Filip A., Bazant L., Mocek H., Taufer J., Maixner V., Dynamic properties of GNSS/ INS based train position locator for signalling applications, COMPRAIL '2002, Lemnos, Greece, 2002, pp.1021-1030.

[6] Filip A., Train real-time position monitoring trials at Czech railways, Structural Integrity and Passenger Safety, WIT press, Great Britain, 1999, pp. 152-166.

[7] Quddus M., Ochieng W., Noland R., Current map-matching algorithms for transport applications: State-of-the art and future research directions, Transportation Research Part C 15, 2007, pp. 312-318.

[8] Jana H., GNSS train position integrity monitoring by the help of discrete PIM algorithms, Journal of Applied Mathematics, vol. 2, no. 3, 2009, pp. 73-79

[9] Saab S., A Map Matching Approach for Train Positioning Part I: Development and Analysis, IEEE Trans. Vehicular Technology, vol. 49, no. 2, 2000, pp. 467-475.

[10] Noronha V., Goodchild M., Map accuracy and location expression in transportation - reality and prospects, Transportation Research Part C 8, 2000, pp. 53-69.

[11] Maixner V., Mocek H., Taufer J., Bazant L., Filip A., The Simulator of Train Position Locator, COMPRAIL '2004, Dresden, Germany, 2004, pp. 477-486.

[12] Meng Y., Chen W., Li Z., Chen Y., Chao J., A Simplified Map Matching Algorithm for In-Vehicle Navigation Unit, Geographic Information Sciences, vol. 8, no. 1, 2002, pp. 24-30.

[13] Arasaratnam I., Haykin S., Cubature Kalman Filters, IEEE Trans. Automatic Control, vol. 54, no. 6, 2009, pp. 1254-1269. 